\title{
User Requirements for a Medical Robotic System: Enabling Doctors to Remotely Conduct Ultrasonography and Physical Examination*
}

\author{
Gerald Stollnberger $^{1}$, Christiane Moser ${ }^{1}$, Manuel Giuliani ${ }^{1}$, Susanne Stadler ${ }^{1}$, \\ Manfred Tscheligi ${ }^{1}$, Dorota Szczesniak-Stanczyk ${ }^{2}$, and Bartlomiej Stanczyk ${ }^{3}$
}

\begin{abstract}
We report the results of a user requirements analysis for a medical robotic system that enables doctors to remotely conduct ultrasonography and physical examination on patients. As there are three different user groups in this scenario - doctors, patients, and assistants - we collected user requirements for all of these groups. This analysis forms a basis for the technical specification of the medical robotic system. To gather the user requirements, we conducted a literature review, observed two examinations of a patient conducted by a doctor, organised four workshops with doctors and patients, and quantified the qualitative data in two online surveys. The most important findings of the requirements analysis are that doctors need accurate kinesthetic, tactile, and audiovisual feedback for a proper diagnosis. They need additional patient data apart from the ultrasonography and physical examination (e.g., olfactory information and skin wetness). Doctors, patients, and assistants all want to have a secure audiovisual communication channel during the whole examination and especially patients have concerns regarding safety of the robot arm and data privacy. We present a list of requirements for doctors, patients, and assistants, and discuss their implications for the technical specifications of the system.
\end{abstract}

\section{INTRODUCTION}

The World Health Statistics Report 2014 [1] by the World Health Organization (WHO) shows that on a world-wide average, a boy and a girl born in 2012 have a life expectancy of 68 years and 73 years, respectively. This means that the global life expectancy is six years higher than it was for children born in 1990. The biggest increase in life expectancy, with an increase of nine years since 1990, was reported from low-income countries, mainly due to a decrease of the mortality of children before their fifth birthday. However, a major difference in mortality rates between rich and poor countries still prevails. The average live expectancy for a boy born in a high-income country is 16 years longer than for a boy born in a low-income country.

One of the main reasons for these numbers is that highincome countries have more well-trained medical personnel and better medical equipment. Broadbent et al. [2] showed that the cost effectiveness in rural areas can be increased by a robot measuring vital signs, which can reduce the monetary burden for health care in low-income countries.

\footnotetext{
*This work was supported by the $7^{\text {th }}$ Framework Program of the European Union, contract number $\mathrm{n}^{\circ} 610902$.

${ }^{1}$ is with the Center for Human-Computer Interaction, Department of Computer Sciences, University of Salzburg, Austria firstname. lastname@sbg.ac.at

2 is with the Department of Cardiology, Medical University of Lublin, Poland dorotaszczesniakstanczyk at umlub.pl

3 is with ACCREA Engineering, Lublin, Poland b.stanczyk at accrea.com
}

Even in rich countries, the availability of doctors can be a problem, for example, at night time or in rural, less densely populated areas. Summarizing, apart from the affordability, the geographical accessibility to well-trained medical doctors is a severe problem.

One possibility to overcome this problem is tele-medicine. The ReMeDi project (Remote Medical Diagnostician ${ }^{4}$ ) is developing and building a medical robot that enables doctors to conduct remote examinations of patients. Fig. 1 and Fig. 2 show the two parts of the system. At the doctor's site (Fig. 1), there is a console that contains the interfaces for communication between the doctor's and the patient's sites and control elements to steer the remote robot. The doctor remotely controls the ReMeDi robot at the patient's site (Fig. 2), which is equipped with sensors and end effectors. Alongside the robot, there is a technical assistant that supports the remote doctor with setting up the robot. The ReMeDi robot supports two types of examination: ultrasonography (the doctor examines the internal organs of the patient by moving an ultrasonic probe over the patient's body), and physical examination (the doctor presses the patient's stomach with her/his hands to feel the stiffness of internal organs and to observe the patient's feedback during the procedure).

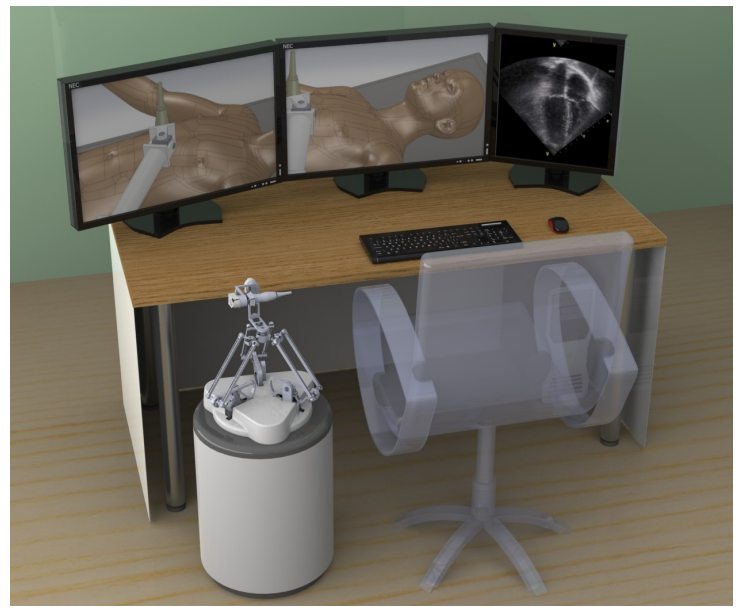

Fig. 1. Visualization of the ReMeDi system at the doctor's location: the doctor has communication interfaces to the patient and the assistant as well as a control console for the remote robot.

In this paper, we report the findings of user requirements studies that we conducted for the ReMeDi system. In order to collect the requirements, we combined qualitative and

\footnotetext{
${ }^{4} \mathrm{http}: / /$ www.remedi-project.eu
} 


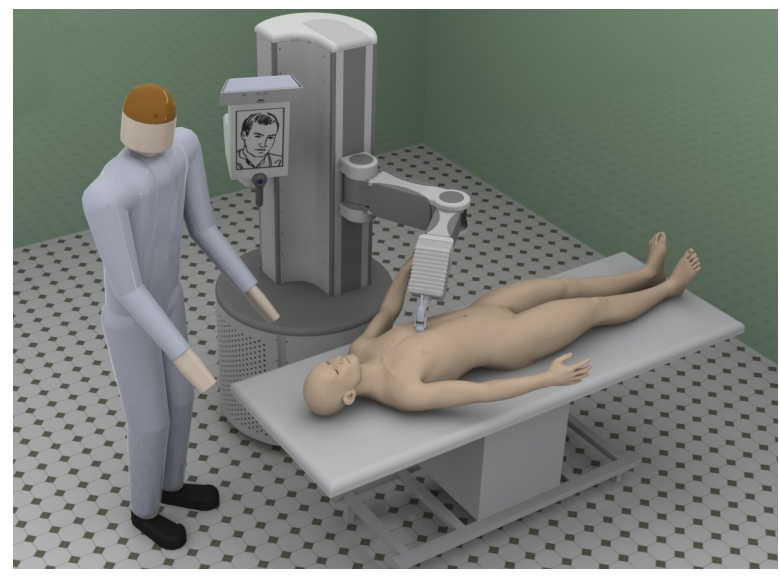

Fig. 2. Visualization of the ReMeDi robot at the patient's location: the robot is remotely controlled by the doctor and executes ultrasonography and palpation on the patient. Robot and patient are accompanied by an assistant, who is responsible for setting up the system and overseeing the examination.

quantitative analysis methods: A literature analysis of requirements for other medical robotic systems, observations of real physical examinations from the patient's perspective to get to know the typical steps executed in a physical examination, two workshops with doctors in which we worked out the requirements they have in a remote medical examination robot, two workshops with patients to figure out which factors are essential for patients to agree to a remote medical examination, and two online surveys to quantify the qualitative results of the workshops. In Section III, we provide details for each of these steps. In Section IV, we list the derived user requirements and discuss them according to their technical feasibility, as well as from a medical and a legal viewpoint in Section V.

\section{BACKGROUND \& RELATED WORK}

Physical examination has its origin in medicine of ancient Greece. Hippocrates was the first person to point out the importance of physical examination for a proper diagnosis. This examination technique was improved by his contemporaries and successive generations of doctors. Before the development of technology-aided examination, physical examination was the only way to conduct a proper medical diagnosis [3]. Although doctors nowadays use advanced medical technology (for example, laboratory diagnostics and imaging techniques), a physical examination is still carried out as the first step when a patient comes in for a consultation. No other medical procedure provides so much information about the human body in such a short time.

The findings of a physical examination can be supplemented by ultrasonography. Medical units execute this examination technique as a test following physical examination. Ultrasonography is usually carried out to examine organs and areas of the human body in which pathologies are expected. A doctor can assess almost all organs and main vessels with ultrasonography without a special preparation of the patient. Since physical examination and ultrasonography are both inexpensive and fast screening techniques that play an important role in almost every diagnosis, these two types of examinations were chosen as areas of interests for the ReMeDi project.

In general, every examination should include an interview, observation, physical examination, and additional examinations and tests (e.g., laboratory tests, ultrasonography and electrocardiologic examination). The existing robotic solutions for remote consultations are, however, often limited to merely teleconferencing, such as the VGO [4] or RP-7 system [5]. Thus, the role of doctors as remote consultants is very limited because the actual physical examination needs to be performed by another doctor. As a consequence, the resulting medical assessment is not based on the specialist's experience, but driven from the interview with patients held by other doctors, who are most likely general practitioners. For an effective diagnosis, a direct physical interaction with the patient is of most importance for a doctor, in order to make use of their expertise and experience.

Robotic systems in medical contexts are seldom controlled over distance, but by an expert physically sitting next to the robot (e.g., [6], [7], and [8]). This obviously does not solve the problem of low geographical availability of doctors. So far, only a few remote medical robot systems have been proposed, for example, for tele-operated echography [9] and for tele-operated ultrasound scanning [10]. Arbeille et al. [11] compared the diagnoses made with either a remote robotic echocardiography system or a manual echocardiography system. They found that the diagnoses were the same in $93 \%$ of cases, which is an indication for the usefulness of remote medical robots. However what is missing, is a system which enables doctors to remotely conduct a complete physical examination beginning from anamnesis, palpation, and ultrasonography to transmitting a diagnosis to the patient.

Other fields of applications for tele-operated medical robots are minimal invasive surgery [12], examining carotid arteries [7], identifying breast cancer [13], monitoring arteries for cardiovascular diseases [6], or taking ultrasound images of patients' arms [14]. A variety of medical robots have been implemented for ultrasound examination, especially for examinations of the abdomen (e.g., [15], [16], and [17])

There are also robotic systems that are used to train doctors in virtual environments. For example, the systems presented in [18] and [19] enable doctors to perform physical examinations on virtual patients. In the training system the doctors interact with a haptic device or a practice dummy to exercise to detect organs and determine their size, shape, consistency, and location.

Finally, the patient's perspective also plays an important role in the development of remote medical robots. So far, only a few user studies looked at the patient's perspective on medical robots. Vilchis et al. [17] report that the volunteer that took part as patient in their study did not feel any discomfort while being diagnosed. Patients of the study described by Delgorge et al. [10] report that they felt confident while interacting with the medical robot system. Martinelli et al. [15] wrote that the 58 patients in their study reported a high satisfaction level, only two were not confident. The 
reason for that was a dysfunction of the haptic device and a computer crash.

Although a lot of research has already been conducted in medical robotic systems, there are no reports of detailed user requirements analyses that take all user groups into account. In particular, the patients' point of view is often unattended or missing. In our user requirement analysis, we consider the needs of doctors, patients, and assistants for the implementation of a remote medical robot that enables doctors to conduct ultrasonography and physical examination remotely. In the following section we describe the process of how we analysed the users' requirements in such a system.

\section{USER REQUIREMENTS ANALYSIS}

Within the ReMeDi project, we follow a user-centred design process [20] for building the remote medical robot, in which the end users - doctors, patients, and assistants are involved. The aim of this process is to take feedback from the end users of the system into account during the whole design and implementation process. The first step in this process is the analysis of user requirements, which we are presenting in this paper.

We began with a literature review in the fields of remote ultrasonography, physical examination, and robotic systems within a medical context. Section II contains parts of this literature review. The most important findings of the review were that there are commercially available devices for remote ultrasonography and for remote medical teleconferencing. Remote palpation, however, has only been done in virtual applications so far.

In the second step, we executed two observations of a health check by a doctor of a patient. The observations were done by one of the authors of this paper as a patient. He received two palpation examinations by the same doctor, the first one during a standard health check, the second one while being ill and having stomach pains. The doctor performed a superficial and a deep palpation in the first check, and only a superficial palpation in the second check in order not to cause pain. The observations allowed us to get a better feeling for the patient's perspective during a physical examination and to generate a list of typically performed examination steps.

In the third step, we organised two workshops with doctors, one in Austria and one in Poland. The workshop participants ( $n=9$, average age 32.11 years, $S D=6.13,5$ female, 4 male) were four cardiologists, two internal medicine doctors, two junior doctors, and one general practitioner, all with expertise in ultrasonography and palpation. The main goals of the workshops were to get a deeper understanding for both examination techniques, as well as to investigate when and why they are used. We also asked for the doctors' opinions about the usage of robotics systems for remote medical examination, using an instant card technique [21]. For a more detailed description of the workshop set-up and results, please refer to [22].

In the fourth step, we organised two workshops with patients, one in Austria and one in Poland. The workshop participants $(n=9$, average age 44.44 years, $S D=19.42,5$ female, 4 male) were healthy at the time of the workshop. The main goals of the workshops were to get a deeper insight into the patients' perspective concerning general examinations and about their opinions and needs regarding examinations executed remotely with a robotic system. In the workshops, we used an adapted version of the robotic toolkit [23], consisting of various robot parts on paper (e.g., heads, arms, legs, etc.), that was used to help participants to express their ideas about the design of a remote medical robot. We also identified under which circumstances patients would agree to a physical examination and to a remote examination in particular. For a more detailed description of the workshop set-up and results, please refer to [24].

Finally, we conducted two online surveys, one with doctors and another one with patients, in order to quantify the insights gained in the workshops. The doctor online survey contained 42 items separated into the categories demographic data, medical issues, attitude towards technology, and system design. In the survey, 53 doctors participated, from which 39 fully completed the questionnaire. The gender distribution amongst the doctors was $49.1 \%$ female, $50.9 \%$ male. As area of expertise, participants reported internal medicine $(22.8 \%)$, radiology $(15.8 \%)$, cardiology $(12.3 \%)$, surgery $(10.5 \%)$, and general medicine $(7.0 \%)$. The patient online survey contained 30 items separated into the categories demographic data, appearance of the robot, and preconditions for agreement to remote examinations. In total, 228 patients participated in the survey. The gender distribution amongst the patients was $71.2 \%$ female and $28.8 \%$ male. As highest level of education, the patients reported graduate degree $(44.8 \%)$, high school diploma(41.2\%), apprenticeship (10.4\%), compulsory school $(3.2 \%)$, and no education $(0.5 \%)$.

By conducting these analysis steps, we gained a deep understanding of the single steps of ultrasonography and physical examination and we quantified the attitudes of doctors and patients towards remote medical examinations and its acceptance. In the following section, we report the user requirements that we derived from these analyses.

\section{USER REQUIREMENTS}

Since the different users of the ReMeDi system-doctors, patients, and assistants-have different needs of the system, we present the requirements for these user groups separately (Section IV-A to Section IV-C). Additionally, we report more general requirements in Section IV-D.

We used affinity diagrams [25] to transfer the requirement analysis data into requirements. Affinity diagrams are a method for organizing ideas, challenges, and solutions into a wall-sized hierarchical diagram. In a first step, we clustered the requirements analysis data into a hierarchical diagram. Afterwards, we sorted the data by requirement categories, mapped the requirements to the different user groups, and validated the requirements with the quantitative data from the online surveys. The requirements are not given in any specific order. 


\section{A. Doctor's User Requirements}

By analysing the data of the different user requirements analysis steps, we identified the following general requirements for doctors:

D1 Doctors want to communicate with the patient during the whole examination using video and audio channels.

D2 Doctors want to have an additional communication channel to the assistant that is separated from communication with the patient and can be put into a private mode in which the patient cannot observe the doctorassistant communication.

D3 Doctors would like to observe local changes of the patient's body temperature and assess wetness of patient's skin.

D4 Olfactory information can be crucial to make a diagnosis in some cases, for example, if the patient is drunk, has vomited, or has diarrhoea.

D5 Regarding the positioning of the robot, doctors want the assistant to position the robot roughly in front of the patient and do the fine-positioning of the system themselves. The doctors have no preference towards a certain input device (e.g., a joystick, 3D mouse, or 3D touch device).

D6 For the doctors, it is essential that the robot is positioned in a way that a visual observation of the patient is possible at all times during the examination.

D7 In addition to ultrasonography and physical examination, the doctors would also like to perform percussion on the patient. In this examination technique, the doctor taps the thorax or abdomen of the patient to determine the underlying structure.

We identified the following ultrasonography requirements for doctors:

D8 Doctors need to position the ultrasound probe with a vertex angle of at least $60^{\circ}$.

D9 Doctors need to see the ultrasound images, preferably in real-time, as the quality of the diagnosis can badly suffer from a long delay in the transmission of the ultrasound images but also in the robot control.

Finally, we identified the following physical examination requirements:

D10 Doctors state that it is not really necessary for them to see their own hands when they palpate a patient by the aid of a robotic system. During a traditional examination, they look at the patients' faces to monitor their reactions. However, the doctors also mentioned that this could increase the necessary training time, due to their unfamiliarity with robots.

D11 It is essential to the doctors that the robotic medical system offers different pressure levels for physical examinations. These should range from very slight, in cases of extreme pain, to hard, which is required for deep palpation.

\section{B. Patient's User Requirements}

The requirements by the patients are clearly driven by the patients' security and privacy concerns when using a remote medical system. We identified the following user requirements for patients:

P1 Patients want to communicate with the doctor during the whole examination process, verbally and visually, preferably keeping eye contact. The patients reported they fear that the social connection with the doctor could suffer, if the doctor is not looking at them.

P2 Patients are strictly against a private communication channel between doctor and assistant.

P3 Patients want to have an assistant present in case of an emergency, which includes technical failures and medical emergencies, for example, a circulatory collapse.

P4 Patients want the robot to have security features. For example, the arm should be back-drivable in an error or panic situation, which means that patients can move the robot arm away at any time during the examination.

P5 Patients want to have a secure data connection between the two modules of the ReMeDi system, in order to secure their privacy.

\section{Assistant's User Requirements}

We identified the following user requirements for assistants:

A1 Assistants need an interface to the robot platform for positioning the system near the patients.

A2 Assistants should be able to transfer additional data to doctors, for example, patient temperature, blood pressure, smell, and skin wetness.

A3 The different end effectors for ultrasonography and physical examination need to be easily detachable by the assistant.

\section{Other Requirements}

The requirements analysis also revealed other requirements, which apply to all user groups:

01 The forces that the robot can apply to the patient's skin need to be restricted.

02 The robot system needs to have emergency buttons for doctors, patients, and assistants that can be pressed in case of technical malfunctions.

\section{DISCUSSION}

The user requirements presented in this paper are a set of needs and preferences of potential users of the ReMeDi system. Some of them might seem to be common sense at first sight. However, we strongly believe that a thorough user requirements analysis and discussion of different requirements is vital for a successful remote medical robotic system. Otherwise, amongst others, we would not have considered the requirement (D4 olfactory information). ReMeDi will enable doctors to remotely conduct ultrasonography and physical examination on patients. In general, our requirement analysis shows that all user groups are positive towards the 
idea to work with a remote robot system. However, the doctors mentioned scepticism about the technical feasibility of such a system. We also found that doctors and patients are often not fully aware of the limitations of current robotic systems, although the majority of them are familiar with new technologies and use them in everyday life.

Therefore, in this section, we discuss how the collected requirements can be transferred into technical specifications and discuss implications for the implementation of these specifications. We look at the technical specifications from three perspectives:

- the technical perspective - to take into account implementation feasibility, patient safety, and system intuitiveness;

- the medical perspective - to look at the necessity of each examination technique and to determine whether certain techniques can be replaced if they are not implementable, or if they can be executed by the assistant; and

- the legal perspective - to resolve questions about system certification and adherence to safety and privacy laws.

Given the project status of ReMeDi at the time of writing this article, the technical perspective has most of our focus. During the workshops and surveys, participants provided a large amount of information about capabilities they would like to see implemented in the ReMeDi system. The support of audiovisual communication between doctor and patient (D1 and P1), as well as between doctor and assistant (D2 and A2), can be easily implemented with current technology.

A challenge that has to be addressed are the "colliding" requirements D2 and P2: doctors would like to have a private communication channel to the assistant, but patients would not like doctors and assistants to communicate about them without them being aware of the content of the communication. The main reason for the private communication channel between doctor and assistant would be the transmission of olfactory information (D4), which can be of vital importance for a correct diagnosis in some cases. At the moment, there are no commercially available technical solutions to recognise and synthesize smells. Therefore, it has to be researched, whether a submission of this information could be transferred by the assistant via a suitable graphical user interface or simply should be openly communicated by the assistant in front of the patient. A suitable solution needs to satisfy both doctor and patient needs similarly.

The observation of local changes of body temperature (D3) is technically achievable but would come with high implementation costs. We have to consider if this additional information for the doctor justifies the costs. A fallback strategy for the implementation of this feature would be to ask the assistant to measure which regions of the patient's body are warmer. The same applies to information about the skin wetness (A2 and D2). Assistants can easily take this measurement without the need for costly hardware, and in contrast to palpation or USG, no specific education is needed.

For the implementation of the positioning of the robot (D5, D6, and A1), there are several state-of-the-art-technologies available. Here, we have to separate rough and fine positioning. The assistant needs to position the robot roughly in front of the patient. For this reason, the ReMeDi partners are studying whether assistants prefer to position the robot manually with an input device such as a game pad or if the robot should position itself automatically. For the fine positioning of the robot arm, the doctor will need a $3 \mathrm{D}$ input device (i.e., a joystick, 3D mouse, or 3D touch device). We will investigate whether force feedback in the device is necessary for a precise positioning. Together with the positioning of the arm, comes the patient need for safety measures allowing them to move the robot arm at all times (P4). The ReMeDi partners will consider the implementation of this feature at the design level of the robot arm. The plan is to build physical safety into the arm itself by including springs and counterbalances into the design of the arm. This also applies to the safe design of the detachable end effectors for ultrasonography and physical examination (A3).

The final technical requirements regard input modalities and feedback channels for remote ultrasonography (D8 and D9) and remote physical examination (D10 and D11). It is crucial for the doctors to have proper kinesthetic and tactile feedback to feel internal organs, lesions, or pathologies during ultrasonographic or physical examination. The information has to be delivered without disturbing delays, which is also complex to address and can become very costly. The same is true on the robot side, which needs to receive the input as closest to real-time as possible and, in addition, is able to apply different pressure levels on the patients' skin for the various examination techniques ultrasonography and superficial or deep palpation. To address these requirements, the ReMeDi project partners are currently building new input devices that provide haptic feedback and an intuitive control of the robot arm. For more details on the technical implementations of these devices, please refer to [26].

Currently, we are only discussing one requirement from the medical perspective. Doctors need to perform percussion with the robotic device (D7). From a medical point of view, percussion can be replaced by ultrasonography in case of abdominal examination. In daily routine, percussion is a fast screening method and all findings have to be confirmed by more detailed imaging techniques, like ultrasonography. Due to the technical difficulty of implementing percussion on a robotic device, the potential costs are not justifiable.

Finally, only one of the requirements is considered from the legal perspective at the moment. Patients want to have a secure data connection for the transmission of their medical data (P5). The ReMeDi partners are currently working together with legal counsellors to implement a secure data connection and data storage that adheres to local and international privacy laws.

\section{CONCLUSION}

Our requirements analysis produced a variety of user requirements, which can contradict each other at times. However, we found that the requirements of the doctors, patients, and assistant fall into the same four categories, 
regardless of user group. Communication (D1, D2, P1, P2) between all user groups is most important. Doctors need to know the status of their patients, patients need to have the reassurance that doctor and assistants are in control of the robot system, and assistants need to have a stable communication channel to get instructions by the doctor.

The second category contains all requirements regarding information (D3, D4, D7, D9, A2). Doctors need to know additional data from their patients, for example, body temperature and skin wetness, which the assistants should prepare and transfer to the doctors.

A third set of requirements falls into the category of robot functionality (D5-D11, P4, A1, A3, 01, O2). Doctors want to have a user interface that provides kinesthetic and tactile feedback for the examination in real-time. Assistants need to position the robot and to detach the different end effectors of the system. Patients want to be able to move the robot away in case of emergencies.

Finally, there are user requirements about safety and privacy (P3-P5, O1, O2). The robot system needs to be built with the safety of the patient in mind. Furthermore, safe data transfer is crucial to guarantee the privacy of patients.

We argue that the full list of user requirements we presented here is also applicable to other remote medical robots, as most of the requirements are not specific to ultrasonography and physical examination. The user requirements falling into the categories robot functionality and safety and privacy are even applicable to medical robots in general.

\section{ACKNOWLEDGEMENTS}

This work was conducted in the frame of the ReMeDi project (funded by the European Union under grant agreement $\mathrm{n}^{o}$ 610902). The Polish part of the research is also financed from the funds for science in the years 2014-2016 granted to a co-financed international project.

\section{REFERENCES}

[1] World Health Organization, "World health statistics 2014, Tech. Rep. WA 900.1, 2014.

[2] E. Broadbent, J. Orejana, H. S. Ahn, J. Xie, P. Rouse, and B. MacDonald, "The cost-effectiveness of a robot measuring vital signs in a rural medical practice," in 24th IEEE Int. Symp. on Robot and Human Interactive Communication (RO-MAN), Aug 2015, pp. 577-581.

[3] V. L. Clark and J. A. Kruse, "Clinical methods: history, physical, and laboratory examinations," JAMA, vol. 264, no. 21, pp. 2808-2809, 1990.

[4] "VGo Communications Webpage," VGo Communications, 2010, (accessed 26.01.2015). [Online]. Available: http://vgocom.com

[5] R. Agarwal, A. W. Levinson, M. Allaf, D. V. Makarov, A. Nason, and L. M. Su, "The roboconsultant: telementoring and remote presence in the operating room during minimally invasive urologic surgeries using a novel mobile robotic interface," Urology, vol. 70, no. 5, pp. 970-974, 2007.

[6] F. Pierrot, E. Dombre, E. Dégoulange, L. Urbain, P. Caron, S. Boudet, G. J., and J.-L. Mégnien, "Hip-pocrate: a safe robot arm for medical applications with force feedback," Medical Image Analysis, vol. 3, no. 3, pp. 285-300, Sept. 1999.

[7] P. Abolmaesumi, S. Salcudean, W.-H. Zhu, M. Sirouspour, and S. DiMaio, "Image-Guided Control of a Robot for Medical Ultrasound," IEEE Trans. on Robotics and Automation, vol. 18, no. 1, pp. 11-23, 2002.

[8] S. Salcudean, W. Zhu, P. Abolmaesumi, S. Bachmann, and P. Lawrence, "A Robot System for Medical Ultrasound," in Proc. of the 9th Int. Symp. of Robotics Research, ISRR 99, 2000, pp. 195-202.
[9] P. Arbeille, J. Ayoub, V. Kieffer, P. Ruiz, B. Combes, A. Coitrieux, P. Herve, S. Garnier, B. Leportz, E. Lefbvre, and F. Perrotin, "Realtime tele-operated abdominal and fetal echography in 4 medical centres, from one expert center, using a robotic arm \& ISDN or satellite link automation," in Proc. of the IEEE Int. Conf. on Automation, Quality and Testing, Robotics, AQTR '08, vol. 1, 2008, pp. 45-46.

[10] C. Delgorge, F. Courrèges, L. A. Bassit, C. Novales, C. Rosenberger, N. Smith-Guerin, C. Brù, R. Gilabert, M. Vannoni, G. Poisson, and V. P., "A tele-operated mobile ultrasound scanner using a light-weight robot," IEEE Trans. on Information Technology in Biomedicine, vol. 9 , no. 1 , pp. 50-58, 2005.

[11] P. Arbeille, R. Provost, K. Zuj, D. Dimouro, and M. Georgescu, "Teles-operated echocardiography using a robotic arm and an internet connection," Ultrasound in Medicine \& Biology, vol. 40, no. 10, pp. 2521-2529, 2014.

[12] G. Watanabe and N. Ishikawa, "da vinci surgical system," Kyobu Geka. The Japanese J. of Thoracic Surgery, vol. 67, no. 8, pp. 686-689, 2014

[13] N. Methil, Y. Shen, D. Zhu, C. Pomeroy, R. Mukherjee, N. Xi, and M. Mutka, "Development of super-media interface for telediagnostics of breast pathology," in Proc. of the IEEE Int. Conf. on Robotics and Automation, 2006, pp. 3911-3916.

[14] F. Conti, J. Park, and O. Khatib, "Interface design and control strategies for a robot assisted ultrasonic examination system," in Experimental Robotics, ser. Springer Tracts in Advanced Robotics, O. Khatib, V. Kumar, and G. Sukhatme, Eds. Springer Berlin Heidelberg, 2014, no. 79, pp. 97-113.

[15] T. Martinelli, J.-L. Bosson, L. Bressollette, F. Pelissier, E. Boidard, J. Troccaz, and P. Cinquin, "Robot-Based Tele-Echography Clinical Evaluation of the TER System in Abdominal Aortic Exploration," $J$. of Ultrasound in Medicine, vol. 26, no. 11, pp. 1611-1616, 2007.

[16] P. Arbeille, A. Capri, J. Ayoub, V. Kieffer, M. Georgescu, and G. Poisson, "Use of a robotic arm to perform remote abdominal telesonography," American J. of Roentgenology, vol. 4, no. 188, pp. 317-322, 2007.

[17] A. Vilchis, J. Troccaz, P. Cinquin, K. Masuda, and F. Pellissier, "A new robot architecture for tele-echography," IEEE Trans. on Robotics and Automation, vol. 19, no. 5, pp. 922-926, Oct. 2003.

[18] S. Ullrich and T. Kuhlen, "Haptic palpation for medical simulation in virtual environments visualization and computer graphics," IEEE Trans. on Visualization and Computer Graphics, vol. 18, no. 4, pp. 617-625, 2012.

[19] S. Yasmin and A. Sourin, "Virtual palpation for medical training in cyberworlds," in Proc. of the Int. Conf. on Cyberworlds, CW'12, Sept. 2012, pp. 207-214.

[20] D. Norman and W. Draper, "User centered system design: New perspectives on human-computer interaction," L. Erlbaum Associates Inc, 1986.

[21] E. Beck, M. Obrist, R. Bernhaupt, and M. Tscheligi, "Instant card technique: How and why to apply in user-centered design," in Proc. of the Tenth Anniversary Conf. on Participatory Design, PDC '08, 2008, pp. 162-165.

[22] G. Stollnberger, C. Moser, E. Beck, C. Zenz, M. Tscheligi, D. Szczesniak-Stanczyk, M. Janowski, W. Brzozowski, R. Blaszczyk, M. Mazur, and A. Wysokinski, "Robotic systems in health care," in Proc. of the 7th Int. Conf. on Human System Interaction, 2014, pp. 276-281.

[23] A. Weiss, D. Wurhofer, M. Lankes, and M. Tscheligi, "Autonomous vs. Tele-operated: How People Perceive Human-Robot Collaboration with HRP-2," in Proc. of the 4th ACM/IEEE Int. Conf. on Human Robot Interaction, HRI '09, 2009, pp. 257-258.

[24] G. Stollnberger, C. Moser, C. Zenz, M. Tscheligi, D. SzczesniakStanczyk, M. Janowski, W. Brzozowski, and A. Wysokinski, "Capturing expected user experience of robotic systems in the health care sector," in Proc. of the Austrian Robotics Workshop, ARW '14, 2014, pp. 42-46.

[25] K. Holtzblatt, J. B. Wendell, and S. Wood, Rapid contextual design: a how-to guide to key techniques for user-centered design. Elsevier, 2004.

[26] A. Peer, M. Buss, B. Stanczyk, D. Szczesniak-Stanczyk, W. Brzozowski, A. Wysokinski, M. Tscheligi, C. A. Avizzano, E. Ruffaldi, L. v. Gool, A. Fossati, K. Arent, J. Jakubiak, and M. Janiak, "Towards a Remote Medical Diagnostican for Medical Examination," in Proc. of the NextMed/MMVR21 Conf., 2014. 